\title{
Estimates for Green's Function in Terms of Asymmetry
}

\section{Tilak Bhattacharya and Allen Weitsman}

\begin{abstract}
Let $\Omega$ be a bounded plane region containing the origin, and having area $\pi$. If $G(z)$ denotes the Green's function for $\Omega$, with pole at 0 , and $G^{*}(r)$ its decreasing rearrangement, then it is shown that $G^{*}(r)<\log (1 / r)-C \alpha^{2}$ for an interval of $r$, where $\alpha$ is the asymmetry of $\Omega$.
\end{abstract}

\section{Introduction}

In this work we continue our study of obtaining bounds on various domain quantities, in terms of asymmetry. For a compact set $\Omega$, in $\mathbb{R}^{n}$, let $V(\Omega)$ denote the volume of $\Omega$ and $B(x, \rho)$ be the ball of radius $\rho$, centered at $x$. Let $\rho$ be such that $V(B(0, \rho))=V(\Omega)$; then we define the asymmetry $\alpha=\alpha(\Omega)$ by

$$
\alpha=\inf _{x} \frac{V(\Omega \backslash B(x, \rho))}{V(\Omega)}, \quad \rho=(V(\Omega) / V(B(x, 1)))^{1 / n} .
$$

It is clear that $\alpha=0$ when $\Omega$ is a ball. In $\mathbb{R}^{2}$, we shall use $A(\Omega)$ to denote the area of $\Omega$.

The works, in [2], [6], [7], [8], and [9] relate asymmetry to various quantities such as the isoperimetric constant, capacity and the first eigenvalue of the Laplacian. In [8], a sharp lower bound for logarithmic capacity, in terms of asymmetry, was deduced by relating capacity to moment of inertia. Such a lower bound was also shown to hold for $p$ capacities of condensers in [2] by very different methods. The analysis in [2] was based on estimates for subcondensers, possessing special geometry, and was also shown to yield a lower bound for logarithmic capacity similar to the one in [8]. For a detailed survey of the field regarding capacity and asymmetry, and the work done in [7], [8] and [9], see [2]. Our effort in this work will be to deduce upper bounds,

\footnotetext{
${ }^{1}$ Mathematics Subject Classification. Primary 31A05, Secondary 30C20.
} 
in terms of asymmetry, for Green's function of a bounded domain in $\mathbb{R}^{2}$. The connection between this and conformal mapping of simply connected regions is obvious, since the Green's function for such an $\Omega$ can be written as $-\log |f|$, where $f$ is the conformal mapping of $\Omega$ onto the unit disk. On the other hand, the estimates on the Green's function are intimately connected to the eigenvalue problem which is still open. We shall give a brief discussion of this in $\S 8$

\section{Notations and the main result}

Given a Borel set $S \in \mathbb{R}^{2}$, let $A(S)$ denote its area and $L(\partial S)$ the perimeter of its boundary $\partial S$. Throughout this work, $\Omega \subset \mathbb{R}^{2}$ will be a bounded domain with boundary $\partial \Omega$ a finite union of rectifiable curves. We will also assume that the origin $0 \in \Omega$. Let $R>0$ be such that $A(\Omega)=\pi R^{2}$; and $G(x), x \in \Omega$, denote Green's function of $\Omega$ with pole at 0 . That is

$$
G(x)=\log \frac{R}{|x|}+h(x), \quad x \in \Omega
$$

where $h(x)$ is harmonic in $\Omega$ and is such that $G(x)$ vanishes continuously on $\partial \Omega$.

Also, let $\Omega^{*}$ be the disk, centered at the origin 0 , having the same area as $\Omega$. Clearly, the radius of $\Omega^{*}$ is $\mathrm{R}$. For $t>0$, we set

$$
F(t)=\{x \in \Omega: G(x)>t\} \text { and } A(t)=A(F(t)) .
$$

For $x \in \Omega^{*}$, the radial function $G^{*}(x)=G^{*}(|x|)$ will denote the symmetric decreasing rearrangement (Schwarz symmetrization) of $G(x)$. It is defined as

$$
G^{*}(x)=G^{*}(|x|)=\inf \left\{t \geq 0: A(t)<\pi|x|^{2}\right\} \quad 0<|x| \leq R
$$

It is well known that $[1 ; \mathrm{p} 60]$,

$$
0 \leq G^{*}(x) \leq \log \frac{R}{|x|}, \quad 0<|x| \leq R, \quad x \in \Omega^{*} .
$$

In this work we determine the effect of asymmetry on (2.4). More precisely, we prove 
Theorem 1 : Let $\Omega \subset \mathbb{R}^{2}$ be a bounded domain with $0 \in \Omega$, and $\alpha=\alpha(\Omega)$ its asymmetry. Let $R>0$ be such that $A(\Omega)=\pi R^{2}$. Then, for every $0<\delta<1$, exists a constant $C=C_{\delta}>0$, depending only on $\delta$, such that

$$
G^{*}(x) \leq \log \frac{R}{|x|}-C \alpha^{2}, \quad 0<|x|<\sqrt{(1-\delta)} R .
$$

We adapt the method developed in [2] to prove Theorem 1 . In what follows, $k$ will stand for a small positive constant to be determined in Sections 3 and 6 . We will assume that

$$
0<k<\delta / 500<10^{-3}
$$

Let $\partial F(t)$ denote the boundary of the set $F(t)$, and $L(\partial F(t))$ be its length . Since $G$ is real analytic in $\Omega \backslash\{0\}$, the gradient $D G$ vanishes only on a discrete set. Thus $\partial F(t)$ is an analytic curve except possibly for countably many $t$ 's, and $A(t)$ is decreasing and continuous.

As in [2], we will consider the following two possibilities.

Case 1 : For all $t$ such that

$$
(1-\delta) \pi R^{2} \leq A(t) \leq(1-\delta / 2) \pi R^{2}
$$

we have

$$
L(\partial F(t))^{2} \geq 4 \pi\left(1+k \alpha^{2}\right) A(t),
$$

that is, asymmetry propagates inwards.

Case 2 : There exists a value $T$ such that

$$
(1-\delta) \pi R^{2} \leq A(T) \leq(1-\delta / 2) \pi R^{2}
$$

and

$$
L(\partial F(T))^{2}<4 \pi\left(1+k \alpha^{2}\right) A(T) .
$$

When this situation occurs, we will say that asymmetry fails to propagate. We may take $\partial F(T)$ to be analytic.

We will also have occassion to use the Bonnesen formulas [11; pp 34]. Let $D$ be a simply connected planar domain bounded by a rectifiable 
Jordan curve. Let $R_{o}$ and $R_{i}$ denote the outradius and inradius of $D$ respectively. Then

and

$$
\begin{gathered}
L(\partial D)^{2}-4 \pi A(D) \geq \pi^{2}\left(R_{o}-R_{i}\right)^{2}, \\
2 \pi R_{o} \leq\left\{L(\partial D)+\sqrt{L(\partial D)^{2}-4 \pi A(D)}\right\},
\end{gathered}
$$

Our strategy will consist in proving the estimate (2.5) in Cases 1 and 2 . We will make considerable use of the coarea formula and the isoperimetric inequality. Our proof in Case 2 will also employ a perturbation result for capacities of condensers. A version was first proven in [2].

Remark 2.1: We will prove Theorem 1 for $R=1$ and $\delta=0.2$. The proof undergoes only minor modifications for $\delta<0.2$ and the full strength of the result in (2.5) for $R \neq 1$ may be recovered by scaling.

We have divided our work as follows. In Section 3, we prove Theorem 1 in Case 1. The proof in Case 2, when $\Omega$ is simply connected, is spread over Sections 4, 5 and 6. In Section 7, we fill in the details of the proof of Theorem 1 when $\Omega$ is multiply connected. Finally, in Section 8, we present a brief discussion regarding the connections of this work to the eigenvalue problem for the Laplacian.

\section{Proof of Theorem 1 in Case 1}

As noted above, for the rest of this work, we take $R=1$ and $\delta=0.2$ in (2.6), (2.7), (2.8) and (2.9).

We now prove Theorem 1, i.e., when asymmetry propagates inwards in the sense of (2.7) and (2.8). By applying the divergence theorem, the coarea formula, and Hölder's inequality we may conclude that outside a set of at most countably many $t^{\prime} s$,

$$
\int_{\partial F(t)}|D G|=2 \pi, \text { and } \frac{d A}{d t}=-\int_{\partial F(t)} \frac{1}{|D G|}, \quad(t<\infty),
$$

and

$$
L(\partial F(t))^{2}=\left(\int_{\partial F(t)} 1\right)^{2} \leq\left(\int_{\partial F(t)}|D G|\right)\left(\int_{\partial F(t)} \frac{1}{|D G|}\right)
$$




$$
=-2 \pi \frac{d A}{d t}
$$

where $A=A(t)$. From (2.7), (2.8), (3.1) and (3.2), it follows that for all $t$ 's such that $0.8 \pi \leq A(t) \leq 0.9 \pi$,

$$
4 \pi\left(1+k \alpha^{2}\right) A(t) \leq-2 \pi \frac{d A}{d t}
$$

Thus

$$
-\frac{1}{2\left(1+k \alpha^{2}\right) A} \frac{d A}{d t} \geq 1
$$

Since $A(t)$ is decreasing and continuous, $-\log A(t)$ is increasing. Thus integrating (3.3), over the interval $[A, 0.9 \pi]$ with $A \geq 0.8 \pi$, we get

$$
t(A)-t(0.9 \pi) \leq \frac{1}{2\left(1+k \alpha^{2}\right)} \log \frac{0.9 \pi}{A} .
$$

Employing the usual isoperimetric inequality (instead of (2.8)) in (3.2), we obtain that

(i) whenever $0<A(t)<0.8 \pi$,

$$
t(A)-t(0.8 \pi) \leq \frac{1}{2} \log \frac{0.8 \pi}{A(t)}
$$

and

(ii) when $0.9 \pi \leq A(t)<\pi$,

$$
t(A)-t(\pi) \leq \frac{1}{2} \log \frac{\pi}{A(t)} .
$$

Recall that $t(\pi)=0$; taking $A=0.9 \pi$ in (3.6) and then adding (3.4) to the resulting inequality in (3.6) we find that for $0.8 \pi \leq A \leq 0.9 \pi$,

$$
\begin{aligned}
t(A) & \leq \frac{1}{2} \log \frac{1}{0.9}+\frac{1}{2} \frac{1}{1+k \alpha^{2}} \log \frac{0.9 \pi}{A} \\
& =\frac{1}{2}\left(\log \frac{\pi}{A}-\frac{k \alpha^{2}}{1+k \alpha^{2}} \log \frac{0.9 \pi}{A}\right)
\end{aligned}
$$

Now take $A=0.8 \pi$ in (3.7). This gives

$$
t(0.8 \pi) \leq \frac{1}{2}\left(\log \frac{1}{0.8}-\frac{k \alpha^{2}}{1+k \alpha^{2}} \log \frac{0.9}{0.8}\right)
$$


Employing this estimate for $t(0.8 \pi)$ in (3.5) results in

$$
\begin{aligned}
t(A) & \leq \frac{1}{2}\left(\log \frac{0.8 \pi}{A}+\log \frac{1}{0.8}-\frac{k \alpha^{2}}{1+k \alpha^{2}} \log \frac{0.9}{0.8}\right) \\
& =\frac{1}{2}\left(\log \frac{\pi}{A}-\frac{k \alpha^{2}}{1+k \alpha^{2}} \log \frac{0.9}{0.8}\right),
\end{aligned}
$$

for $0<A<0.8 \pi$.

For $0<A<0.85 \pi$ we may then deduce the following estimate from (3.7) and (3.8), namely,

$$
t(A) \leq \frac{1}{2}\left(\log \frac{\pi}{A}-\frac{k \alpha^{2}}{1+k \alpha^{2}} \log \frac{0.9}{0.85}\right) .
$$

Let $B(0, r)$ denote the disk with $\pi r^{2}=A(t)$. Noting that $t(A)=G^{*}(r)$, we have

$$
G^{*}(r) \leq \log \frac{1}{r}-\frac{k \alpha^{2}}{1+k \alpha^{2}} \log \frac{0.9}{0.85},
$$

for $0<r<\sqrt{0.85}$. Theorem 1 will follow once a value for $k$ is chosen.

The remainder of the proof is devoted to Theorem 1 in Case 2, i.e., when (2.9) and (2.10) hold. We will make some preliminary reductions before presenting the proof. A part of the effort will be invested in describing the geometry of the level sets $F(t)$ involved in this case. The proof will follow from a perturbation result for capacities of condensers.

\section{Preliminary Reductions}

We will first assume that $\Omega$ is simply connected. The analysis continues to apply with minor modifications in the event that $\Omega$ is multiply connected. These details are presented in Section 7 .

The following lemma, though not difficult to prove, is essential for the construction of a condenser with special geometry. A lower bound for its 2-capacity will be key to the proof of Theorem 1 in Case 2.

Lemma 4.1 : Either there exists a $t=t_{0}>0$ with

$$
A\left(t_{0}\right)>\pi\left(1-k \alpha^{2}\right)
$$


and

$$
L\left(\partial F\left(t_{0}\right)\right)^{2}<4 \pi(1.01) A\left(t_{0}\right)
$$

or Theorem 1 holds.

Proof : Suppose that (4.2) were false for all $t$ 's such that (4.1) holds, i.e.,

$$
L(\partial F(t))^{2} \geq 4 \pi(1.01) A(t), \text { whenever } \pi\left(1-k \alpha^{2}\right)<A(t)<\pi .
$$

Then using the above mentioned inequality, in (3.2), we get for $\pi\left(1-k \alpha^{2}\right) \leq A(t) \leq \pi$

$$
-\frac{1}{2(1.01) A} \frac{d A}{d t} \geq 1 .
$$

Integrating (4.3) over the interval $\left[\pi\left(1-k \alpha^{2}\right), \pi\right]$ we get that

$$
t\left(\pi\left(1-k \alpha^{2}\right)\right) \leq \frac{1}{2(1.01)} \log \frac{1}{1-k \alpha^{2}}
$$

Now employing the usual isoperimetric inequality in (3.2) and integrating over the interval $\left[A, \pi\left(1-k \alpha^{2}\right)\right]$ ( for example, see $\left.(3.5)\right)$ we find that

$$
t(A)-t\left(\pi\left(1-k \alpha^{2}\right)\right) \leq \frac{1}{2} \log \frac{\pi\left(1-k \alpha^{2}\right)}{A} .
$$

Adding (4.4) and (4.5) and recalling (2.6) we get that for $0<A<$ $0.85 \pi$

$$
t(A) \leq \frac{1}{2}\left(\log \frac{\pi}{A}-\frac{0.01}{1.01} \log \frac{1}{1-k \alpha^{2}}\right)
$$

which again implies Theorem 1 .

We now continue the proof of Theorem 1 under the assumption that there exists a value of $t$ such that both (4.1) and (4.2) hold. In what follows, we take $t_{0}$ to always denote such a value and this will stay fixed throughout. Let $T$ be as in (2.9) and (2.10). It readily follows from (2.6) and (2.9) that

$$
T>t_{0}
$$

We now describe the geometry of the set $F(T)$. Since $G(x)$ has only one singularity, the level sets $F(t)$, for all $t$, are simply connected by 
the maximum principle. With $A(t)=A(F(t))$, set $R=\sqrt{A\left(t_{0}\right) / \pi}$ and $\rho=\sqrt{A(T) / \pi}$; it is easily seen from (2.6), (2.9) and (4.1) that

$$
\sqrt{0.8} \leq \rho \leq \sqrt{0.9} \text { and } \sqrt{0.999}<R<1 .
$$

It follows from the Bonnesen formulas in (2.11) - (2.13) that $\partial F(T)$ is contained between two circles $C_{1}=\left\{x:\left|x-x_{o}\right|=R_{o}\right\}$ and $C_{2}=\{x$ : $\left.\left|x-x_{i}\right|=R_{i}\right\}$, where $R_{o}$ and $R_{i}$ are the outradius and the inradius of $F(T)$ respectively. Thus,

$$
0 \leq R_{o}-R_{i} \leq 2 \sqrt{\frac{k A(T)}{\pi}} \alpha=2 \sqrt{k} \alpha \rho \leq 2 \sqrt{k} \alpha,
$$

$$
\begin{aligned}
R_{o} & \leq \frac{L(\partial F(T))}{2 \pi}+\sqrt{\frac{k A(T)}{\pi}} \alpha \leq\left(\sqrt{1+k \alpha^{2}}+\sqrt{k} \alpha\right) \sqrt{\frac{A(T)}{\pi}} \\
& \leq(1+2 \sqrt{k} \alpha) \sqrt{\frac{A(T)}{\pi}}=(1+2 \sqrt{k} \alpha) \rho \\
R_{i} & \geq \frac{L(\partial F(T))}{2 \pi}-\sqrt{\frac{k A(T)}{\pi}} \alpha \geq(1-\sqrt{k} \alpha) \sqrt{\frac{A(T)}{\pi}}=(1-\sqrt{k} \alpha) \rho,
\end{aligned}
$$

where we have used (2.10) to calculate $R_{o}$ and the usual isoperimetric inequality to estimate $R_{i}$. Also, from (4.7),

$$
\left|x_{o}-x_{i}\right| \leq R_{o}-R_{i} \leq 2 \sqrt{\frac{k A(T)}{\pi}} \alpha=2 \sqrt{k} \rho \alpha \leq 2 \sqrt{k} \alpha .
$$

We set

$$
\varepsilon=6 \sqrt{k} \alpha
$$

Now, from (4.7), (4.8), (2.6) and (2.9), it follows that $\left|x_{o}-x_{i}\right|<R_{i}$. Since $x_{i} \in F(T)$, we have that $x_{o} \in F(T)$. Thus it is easily reasoned from (4.7) that $F(T) \subset B\left(x_{i}, \bar{R}_{o}\right)$ with

$$
\bar{R}_{o} \leq 2 R_{o}-R_{i} \leq(1+5 \sqrt{k} \alpha) \rho \leq 1.11 .
$$

We will now use (2.12) to estimate the outradius $\hat{R}_{o}$ of $F\left(t_{0}\right)$. It follows from (4.1) and (4.2) that

$$
\hat{R}_{o} \leq(\sqrt{1.01}+\sqrt{0.01}) \sqrt{\frac{A\left(t_{0}\right)}{\pi}} \leq 1.11 R .
$$


If we use (2.6), (2.9), (4.1) and (4.7), we find that $F\left(t_{0}\right) \subset B\left(x_{i}, \tilde{R}_{o}\right)$ where

$$
\tilde{R}_{o} \leq 2 \hat{R}_{o}-R_{i} \leq 2.22 R-(1-\sqrt{k} \alpha) \rho \leq 1.40 .
$$

The estimate in (4.11) follows quite easily from the observation that $B\left(x_{i}, R_{i}\right) \subset F(T) \subset F\left(t_{0}\right) \subset B\left(x_{i}, \tilde{R}_{o}\right)$. Again, with $x_{i}$ as in (4.8), it follows from (4.1), (2.6) and (1.1) that if

$$
\beta=\frac{A\left(F\left(t_{0}\right) \backslash B\left(x_{i}, R\right)\right)}{A\left(t_{0}\right)}, \quad R=\sqrt{A\left(t_{0}\right) / \pi},
$$

then

$$
\begin{aligned}
\beta & \geq \frac{A\left(\Omega \backslash B\left(x_{i}, R\right)\right)-A\left(\Omega \backslash F\left(t_{0}\right)\right)}{\pi} \\
& \geq \frac{A\left(\Omega \backslash B\left(x_{i}, 1\right)\right)-A\left(\Omega \backslash F\left(t_{0}\right)\right)}{\pi} \\
& \geq \alpha-k \alpha^{2} \\
& \geq \alpha / 2
\end{aligned}
$$

Let $\Gamma$ denote the condenser $\Gamma\left(\bar{F}(T), \mathbb{R}^{2} \backslash \underline{F}\left(t_{0}\right)\right)$ whose inner set is $\bar{F}(T)$ and whose outer set is $\mathbb{R}^{2} \backslash F\left(t_{0}\right)$. Here $\bar{S}$ stands for the closure of a set $S$. The following then summarizes the description of the geometry of the condenser $\Gamma$. From (2.6), (2.9) and (4.7) - (4.11), we see that

(i) $B\left(x_{i}, R_{i}\right) \subset F(T) \subset B\left(x_{i}, \bar{R}_{o}\right)$,

(ii) $F\left(t_{0}\right) \subset B\left(x_{i}, \tilde{R}_{o}\right)$,

(iii) $\quad \bar{R}_{o}-R_{i} \leq 4 \sqrt{k} \alpha \rho \leq 4 \sqrt{k} \alpha \leq \varepsilon$, where $\varepsilon=6 \sqrt{k} \alpha$,

(iv) $4 / 5 \leq 1-6 \sqrt{k} \alpha \leq R_{i} / \bar{R}_{o} \leq \rho / \bar{R}_{o}$,

(v) $\quad 4 / 7 \leq(1-\sqrt{k} \alpha) \rho / 1.40 \leq R_{i} / \tilde{R}_{o}$

$$
\leq \rho / \tilde{R}_{o} \leq \rho / R \leq \sqrt{\frac{100}{111}}
$$

(vi) $\beta=A\left(F\left(t_{0}\right) \backslash B\left(x_{i}, R\right)\right) / A\left(t_{0}\right) \geq \alpha / 2$.

(vii) $R_{i} \leq \rho \leq \bar{R}_{o}$ and $R \leq \hat{R}_{o} \leq \tilde{R}_{o}$.

Let $\operatorname{Cap}(\Gamma)$ be the 2-capacity of $\Gamma$, i.e.,

$$
\operatorname{Cap}(\Gamma)=\operatorname{Cap}_{2}(\Gamma)=\inf _{w} \int_{\mathbb{R}^{2}}|D w|^{2} d x d y,
$$


where $w$ is absolutely continuous on $\mathbb{R}^{2}$ and takes the value 1 on $\bar{F}(T)$ and 0 on $\mathbb{R}^{2} \backslash F\left(t_{0}\right)$. The minimizer $v$ is harmonic, in $F\left(t_{0}\right) \backslash \bar{F}(T)$, and is given by $\left(G-t_{0}\right) /\left(T-t_{0}\right)$. With $\varepsilon, x_{i}, \beta$ as above, and $v=$ $\left(G-t_{0}\right) /\left(T-t_{0}\right)$ we now prove a perturbation result for the 2-capacity of the condenser $\Gamma$.

\section{$5 \quad$ A general perturbation result.}

Before proving the perturbation result for 2-capacities of condensers, we will first describe a new type of symmetrization first introduced in [2]. The version, we use in this work, differs from the one in [2] in that it is designed to handle inward propagation of asymmetry. In the current situation we redistribute the outer set instead of the inner set as was done in [2]. We first describe this symmetrization in a general setting and then apply this to obtain a perturbation type result for the 2-capacity of condensers with special geometry ( see the last paragraph of $\S 4)$.

Let $O$ and $Q$ be two bounded open sets in $\mathbb{R}^{2}$. We assume that (i) the origin 0 lies in $O$, (ii) $\bar{O} \subset Q$, and (iii) $\partial O$ and $\partial Q$ are unions of finitely many Lipschitz curves. The requirement in (i) is purely for the ease of presentation of details and bears no relation to the assumption in Theorem 1. Let $\rho=\sqrt{A(O) / \pi}$ and $R=\sqrt{A(Q) / \pi}$.

For each $\theta \in(-\pi, \pi]$, let $J(\theta)=\left\{r e^{i \theta}: 0 \leq r\right\}$ be the ray from the origin making an angle $\theta$ with the positive $x$-axis. For a given value of $\theta$, let

$$
J(\theta) \cap Q=\left[r_{0}, r_{1}(\theta)\right) \cup_{j \geq 1}\left(r_{2 j}(\theta), r_{2 j+1}(\theta)\right),\left(r_{0}=0\right),
$$

the intervals being pairwise disjoint. We now introduce the following quantities necessary to give a redistribution of the area of $Q$ relative to $B(0, R)$.

Set

$$
\begin{aligned}
s(\theta) & =\sup \left\{r: r e^{i \theta} \in J(\theta) \cap O\right\} \\
t(\theta) & =\inf \left\{r: r e^{i \theta} \in J(\theta) \cap \partial Q\right\} \\
& =\sup \{r:[0, r) \subset J(\theta) \cap Q\}, \\
\hat{s}(\theta) & =\sup \left\{r: r e^{i \theta} \in J(\theta) \cap O, r<t(\theta)\right\}, \\
\hat{t}(\theta) & =\inf \left\{r: r e^{i \theta} \in J(\theta) \cap \partial Q, r>s(\theta)\right\}
\end{aligned}
$$




$$
\begin{aligned}
& =\sup \{r:[s(\theta), r) \subset Q\}, \\
N & =\left\{r e^{i \theta} \in Q^{c}: s(\theta)>t(\theta), t(\theta)<r<s(\theta)\right\} \\
E & =\{\theta: J(\theta) \cap N \neq \emptyset\}=\{\theta: s(\theta)>t(\theta)\} .
\end{aligned}
$$

Here $Q^{c}$ stands for the complement of $Q$. It is useful to observe that $s(\theta) \geq \hat{s}(\theta), \hat{t}(\theta) \geq t(\theta)$ with equality iff $s(\theta)<t(\theta)$. We now distinguish two possibilities in the redistribution of $Q$.

Case A: Suppose first that $\hat{t}(\theta) \geq R$. Define $\xi(\theta) \geq \hat{t}(\theta)$ by

$$
\xi(\theta)^{2}=\hat{t}(\theta)^{2}+\sum_{i \in I}\left(r_{2 i+1}(\theta)^{2}-r_{2 i}(\theta)^{2}\right)
$$

where $I=\left\{i: r_{2 i}(\theta) \geq \hat{t}(\theta)\right\}$. Then $\xi(\theta) \geq R$.

Case B: If $\hat{t}(\theta)<R$, we distinguish two subcases to define $\xi(\theta) \geq \hat{t}(\theta)$ and $\lambda(\theta) \geq R$.

(i) If $R e^{i \theta} \in J(\theta) \cap Q$, i.e., $r_{2 j}(\theta)<R<r_{2 j+1}(\theta)$ for some $j$, then

$$
\xi(\theta)^{2}=\hat{t}(\theta)^{2}+R^{2}-r_{2 j}(\theta)^{2}+\sum_{i \in L}\left(r_{2 i+1}(\theta)^{2}-r_{2 i}(\theta)^{2}\right),
$$

where $L=\left\{i: \hat{t}(\theta) \leq r_{2 i}(\theta)<r_{2 i+1}(\theta) \leq r_{2 j}(\theta)\right\}$, and

$$
\lambda(\theta)^{2}=r_{2 j+1}(\theta)^{2}+\sum_{i \in M}\left(r_{2 i+1}(\theta)^{2}-r_{2 i}(\theta)^{2}\right),
$$

where $M=\left\{i: r_{2 i}(\theta) \geq r_{2 j+1}(\theta)\right\}$. Then $\xi(\theta) \leq R$ and $\lambda(\theta)>R$.

(ii) If $R e^{i \theta} \in J(\theta) \backslash Q$, we set

$$
\xi(\theta)^{2}=\hat{t}(\theta)^{2}+\sum_{i \in L^{\prime}}\left(r_{2 i+1}(\theta)^{2}-r_{2 i}(\theta)^{2}\right),
$$

where $L^{\prime}=\left\{i: \hat{t}(\theta) \leq r_{2 i}(\theta)<r_{2 i+1}(\theta)<R\right\}$, and

$$
\lambda(\theta)^{2}=R^{2}+\sum_{i \in M^{\prime}}\left(r_{2 i+1}(\theta)^{2}-r_{2 i}(\theta)^{2}\right)
$$

where $M^{\prime}=\left\{i: r_{2 i}(\theta) \geq R\right\}$. Then $\xi(\theta) \leq R$ and $\lambda(\theta) \geq R$.

Now suppose that $0<R_{i} \leq \rho \leq \bar{R}_{o}$, and $0<R \leq \tilde{R}_{o}$ are such that $B\left(0, R_{i}\right) \subset O \subset \bar{O} \subset B\left(0, \bar{R}_{o}\right)$ and $\bar{Q} \subset B\left(0, \tilde{R}_{o}\right)$. Then (5.1)-(5.6) 
imply

(i) $\quad R_{i} \leq \hat{s}(\theta) \leq s(\theta) \leq \bar{R}_{o}$,

(ii) $\quad R_{i} \leq t(\theta) \leq \hat{t}(\theta) \leq \xi(\theta) \leq \tilde{R}_{o}$

(iii) $s(\theta)<\hat{t}(\theta) \leq \xi(\theta)$

(5.7) (iv) $\quad R_{i} \leq \hat{s}(\theta)<t(\theta)<s(\theta) \leq \bar{R}_{o}, \theta \in E$,

(v) if $\hat{t}(\theta)<R$ then $\xi(\theta)<R$ and $\lambda(\theta) \geq R$,

(vi) if $\hat{t}(\theta) \geq R$ then $\xi(\theta) \geq R$,

(vii) $\quad R_{i} \leq \rho \leq \min \left(R, \bar{R}_{o}\right)$ and $R \leq \tilde{R}_{o}$.

(viii) $\hat{s}(\theta) \leq s(\theta)$ and $t(\theta) \leq \hat{t}(\theta)$ with equality iff $s(\theta)<t(\theta)$.

It is helpful to note that $R_{i} \leq \inf _{\theta}\{\sup \{r:[0, r) \subset J(\theta) \cap O\}\}$ and $\bar{R}_{o} \geq \sup _{\theta} s(\theta)$.

Based on (5.1)-(5.7), we now make some easy but useful observations. Suppose that $\beta=A(Q \backslash B(0, R)) / A(Q)$. Then by consideration of $B(0, R) \backslash Q,(5.7)(\mathrm{v})$ and (vi),

$$
\begin{aligned}
0<2 \pi R^{2}\left(\beta-\frac{A(N \cap B(0, R))}{\pi R^{2}}\right) & \leq \int_{\{\xi(\theta) \leq R\}} R^{2}-\xi(\theta)^{2} d \theta \\
& \leq 2 \pi R^{2} \beta
\end{aligned}
$$

By consideration of $Q \backslash B(0, R),(5.7)$ (v) and (vi),

$$
\begin{aligned}
2 \pi R^{2} \beta & \leq \int_{\{\xi(\theta) \geq R\}} \xi(\theta)^{2}-R^{2} d \theta+\int_{\{\hat{t}(\theta)<R\}} \lambda(\theta)^{2}-R^{2} d \theta \\
& \leq 2 \pi R^{2}\left(\beta+\frac{A(N \backslash B(0, R))}{\pi R^{2}}\right) .
\end{aligned}
$$

Subtracting (5.8) from (5.9), we get

$$
0 \leq \int_{-\pi}^{\pi} \xi(\theta)^{2}-R^{2} d \theta+\int_{\{\hat{t}(\theta)<R\}} \lambda(\theta)^{2}-R^{2} d \theta \leq 2 A(N)
$$

By adding (5.8) and (5.9), and employing (5.7) (v), we get

$$
\int_{-\pi}^{\pi}\left|\xi(\theta)^{2}-R^{2}\right| d \theta \leq 4 \pi R^{2}\left(\beta+\frac{A(N)}{\pi R^{2}}\right) .
$$

Also define $\mu$ and $\bar{\mu}$ by

$$
2 \pi R^{2} \mu=\int_{-\pi}^{\pi} \xi(\theta)^{2}-R^{2} d \theta, \quad 2 \pi R^{2} \bar{\mu}=\int_{-\pi}^{\pi} s(\theta)^{2}-\rho^{2} d \theta
$$


By (5.1), (5.7) (v) and (5.10),

$$
\mu \leq \frac{A(N)}{\pi R^{2}} \text { and } \bar{\mu} \geq 0
$$

We now prove a perturbation result for 2-capacity.

Let $O$ and $Q$ be as before, satisfying $B\left(0, R_{i}\right) \subset O \subset \bar{O} \subset B\left(0, \bar{R}_{o}\right)$, and

$O \subset \bar{O} \subset Q \subset \bar{Q} \subset B\left(0, \tilde{R}_{o}\right)$. Let $\rho=\sqrt{A(O) / \pi}$ and $R=\sqrt{A(Q) / \pi}$.

Then

$0<R_{i} \leq \rho \leq \bar{R}_{o}$ and $R \leq \tilde{R}_{o}$. Assume further that

(i) for a fixed $\varepsilon, 0<\varepsilon \leq 1 / 2,(1-\varepsilon) \bar{R}_{o}<R_{i} \leq \rho \leq \bar{R}_{o}$,

(ii) $1 / 2 \leq \min \left\{R_{i} / \tilde{R}_{o}, R_{i} / \bar{R}_{o}, \rho / R\right\}$

$$
\leq \max \left\{R_{i} / \tilde{R}_{o}, R_{i} / \bar{R}_{o}, \rho / R\right\} \leq 1
$$

(iii) for a fixed $\delta, 0<\delta \leq 1 / 2,1 / 4 \leq(\rho / R)^{2} \leq 1 /(1+\delta)<1$.

Let $\Gamma$ denote the condenser $\Gamma\left(\bar{O}, \mathbb{R}^{2} \backslash Q\right)$; set

$$
I=\operatorname{Cap}_{2}(\Gamma)=\inf _{u} \int_{Q \backslash \bar{O}}|D u|^{2} d x d y
$$

where $u$ is absolutely continuous and takes the value 1 on $\mathbb{R}^{2} \backslash Q$ and 0 on $\bar{O}$. Let $v$ denote the minimizer. Then $v$ is harmonic in $Q \backslash \bar{O}$ and assumes the appropriate boundary values. Let $\beta=A(Q \backslash B(0, R)) / A(Q)$, where $R=\sqrt{A(Q) / \pi}$ (see line preceding (5.8)). We prove

Lemma 5.1: Let $O, Q, \rho, R, R_{i}, \bar{R}_{o}, \tilde{R}_{o}, \beta, \varepsilon$ and $v$ be as described above. Assume that (5.14) holds. Then for all sufficiently small $\varepsilon>0$, we have

$I=\operatorname{Cap}_{2}(\Gamma)=\int_{Q \backslash \bar{O}}|D v|^{2} d x d y \geq \frac{2 \pi}{\log R / \rho}+K_{0} \beta^{2}-K_{1} \varepsilon^{2}-K_{2} \varepsilon \beta$,

where $K_{0}, K_{1}$ and $K_{2}$ are positive constants depending only on $\delta$.

Proof: Throughout this proof $C_{j}$ 's will denote positive constants which are either absolute or depend only on $\delta$. We shall employ the symmetrization described above with the same notations as in (5.1)-(5.6). 
From (5.7) and (5.14) we see that

(i) $0<t(\theta)-\hat{s}(\theta) \leq \bar{R}_{o}-R_{i} \leq \varepsilon \bar{R}_{o}, \theta \in E$,

$(5.15)(i i) \quad(1 / e)^{2}<1 / 4 \leq \min \left(s(\theta)^{2} / \xi(\theta)^{2}, \rho^{2} / R^{2}, R_{i}^{2} / \bar{R}_{o}^{2}, R_{i}^{2} / \tilde{R}_{o}^{2}\right)$,

(iii) $\left|\rho^{2}-s(\theta)^{2}\right| \leq 2 \varepsilon \bar{R}_{o}^{2}$,

(iv) $1-\varepsilon \leq R_{i} / \bar{R}_{o} \leq s(\theta) / \bar{R}_{o} \leq 1$.

Now

$$
\begin{aligned}
I & =\int_{Q \backslash \bar{O}} v_{r}^{2}+\frac{1}{r^{2}} v_{\theta}^{2} r d r d \theta \\
& \geq \int_{Q \backslash \bar{O}} v_{r}^{2} r d r d \theta \\
& \geq \int_{-\pi}^{\pi}\left(\inf _{z} \int_{J(\theta) \cap\{Q \backslash \bar{O}\}} z_{r}^{2} r d r\right) d \theta
\end{aligned}
$$

where the infimum is taken over $z=z(r, \theta)$ such that $z=1$ on $J(\theta) \cap$ $\left(\mathbb{R}^{2} \backslash Q\right)$ and $z=0$ on $J(\theta) \cap \bar{O}$. The minimizer $\bar{z}$ satisfies the one variable Euler equation $\left(r \bar{z}^{\prime}\right)^{\prime}=0$ in $J(\theta) \cap\{Q \backslash \bar{O}\}$. We will estimate $I$ by employing the symmetrization described above and obtaining a lower bound for the inner integral on the right hand side of (5.16). We do this by first solving for $\bar{z}$ from the o.d.e. over the disjoint intervals $(s(\theta), \hat{t}(\theta))$ and $(\hat{s}(\theta), t(\theta))$, the latter occuring whenever $s(\theta)>t(\theta)$, i. e., when $\theta \in E$ (see (5.1)). Note that $\bar{z}$ vanishes on the left end points of these intervals and takes the value 1 on the right end points. Also see (5.7). Thus a lower bound for $I$ is obtained by calculating the inner integral for this function $\bar{z}$ over the above mentioned intervals. Recalling the definition of $E$ from (5.1), it follows from (5.16), (5.7) and (5.1) that

$$
\begin{aligned}
I & \geq \int_{-\pi}^{\pi} \frac{1}{\log (\hat{t}(\theta) / s(\theta))} d \theta+\int_{E} \frac{1}{\log (t(\theta) / \hat{s}(\theta))} d \theta \\
& \geq \int_{-\pi}^{\pi} \frac{1}{\log (\xi(\theta) / s(\theta))} d \theta+\int_{E} \frac{1}{\log (t(\theta) / \hat{s}(\theta))} d \theta .
\end{aligned}
$$

If the second integral, on the right hand side of (5.17), is larger than $4 \pi / \log (R / \rho)$, then Lemma 5.1 follows trivially from (5.15) (ii). Otherwise,

$$
\int_{E} \frac{1}{\log (t(\theta) / \hat{s}(\theta))} d \theta \leq \frac{4 \pi}{\log (R / \rho)}
$$


But $\log (t(\theta) / \hat{s}(\theta)) \leq(t(\theta) / \hat{s}(\theta)-1)$; it then follows from (5.15) (i), (5.7) (iv), (5.15) (ii), (5.14) (iii) that

$$
\operatorname{meas}_{\theta} E \leq \frac{16 \pi \varepsilon}{\log (1+\delta)}=C_{1} \varepsilon
$$

Recalling the definition of $N$ from (5.1), (5.7) (iv) and (5.14) (i) yield

$$
A(N) \leq \frac{32 \pi \varepsilon^{2}}{\log (1+\delta)} \bar{R}_{o}^{2}=C_{2} \varepsilon^{2} \bar{R}_{o}^{2}
$$

Now from (5.17),

$$
\begin{aligned}
I & \geq \int_{-\pi}^{\pi} \frac{1}{\log (\xi(\theta) / s(\theta))} d \theta \\
& =2 \int_{-\pi}^{\pi} \frac{-1}{\log \left(s(\theta)^{2} / \xi(\theta)^{2}\right)} d \theta .
\end{aligned}
$$

To estimate (5.19) we note that the function $f(x)=-1 / \log x$ satisfies

$$
\begin{array}{ll}
\text { (i) } & f(x)>0,(0<x<1), \\
\text { (ii) } & f^{\prime}(x)>0,(0<x<1), \\
\text { (iii) } & f^{\prime \prime}(x)>0,\left(1 / e^{2}<x<1\right) .
\end{array}
$$

We shall use (5.20) in the form

$$
f(x)=f(\bar{x})+f^{\prime}(\bar{x})(x-\bar{x})+\frac{f^{\prime \prime}(\zeta)}{2}(x-\bar{x})^{2},
$$

for some $\zeta \in(x, \bar{x})$ or $(\bar{x}, x)$. From (5.7) (iii) and (5.14) (iii), it follows that $s(\theta) / \xi(\theta)<1$ and $1 / 2 \leq \rho / R \leq 1 / \sqrt{1+\delta}<1$. Then with $\bar{x}=\rho^{2} / R^{2}$, it follows from (5.19), (5.20) and (5.21) that

$$
\begin{aligned}
I-\frac{2 \pi}{\log R / \rho} & \geq 2 \int_{-\pi}^{\pi} \frac{-1}{\log \left(s(\theta)^{2} / \xi(\theta)^{2}\right)}+\frac{1}{\log \left(\rho^{2} / R^{2}\right)} d \theta \\
& \geq 2 f^{\prime}\left(\rho^{2} / R^{2}\right) \int_{-\pi}^{\pi}\left(\frac{s(\theta)^{2}}{\xi(\theta)^{2}}-\frac{\rho^{2}}{R^{2}}\right) d \theta \\
& +C_{3} \int_{-\pi}^{\pi}\left(\frac{s(\theta)^{2}}{\xi(\theta)^{2}}-\frac{\rho^{2}}{R^{2}}\right)^{2} d \theta
\end{aligned}
$$

The positive constant $C_{3}$, in (5.22), results from the fact that $(5.15)$ (ii) implies that $\zeta \geq \min \left(s(\theta)^{2} / \xi(\theta)^{2}, \rho^{2} / R^{2}\right) \geq 1 / 4>1 / e^{2}$. 
Next we estimate the quantities

$$
B=\int_{-\pi}^{\pi} \frac{s(\theta)^{2}}{\xi(\theta)^{2}}-\frac{\rho^{2}}{R^{2}} d \theta, \text { and } D=\int_{-\pi}^{\pi}\left(\frac{s(\theta)^{2}}{\xi(\theta)^{2}}-\frac{\rho^{2}}{R^{2}}\right)^{2} d \theta
$$

We may rewrite $B$ as

$$
\begin{aligned}
B & =\int_{-\pi}^{\pi} s(\theta)^{2}\left(\frac{1}{\xi(\theta)^{2}}-\frac{1}{R^{2}}\right)+\frac{s(\theta)^{2}-\rho^{2}}{R^{2}} d \theta \\
& =\int_{-\pi}^{\pi}\left(s(\theta)^{2}-\rho^{2}\right)\left(\frac{1}{\xi(\theta)^{2}}-\frac{1}{R^{2}}\right) \\
& +\rho^{2}\left(\frac{1}{\xi(\theta)^{2}}-\frac{1}{R^{2}}\right)+\frac{s(\theta)^{2}-\rho^{2}}{R^{2}} d \theta .
\end{aligned}
$$

By (5.12) and (5.13),

$$
\int_{-\pi}^{\pi} \frac{s(\theta)^{2}-\rho^{2}}{R^{2}} d \theta \geq 0
$$

Also by (5.15) (iii), (5.7) (ii), (5.14) (ii) and (5.11),

$$
\begin{aligned}
\left|\int_{-\pi}^{\pi}\left(s(\theta)^{2}-\rho^{2}\right)\left(\frac{1}{\xi(\theta)^{2}}-\frac{1}{R^{2}}\right) d \theta\right| & \leq \int_{-\pi}^{\pi}\left|s(\theta)^{2}-\rho^{2}\right| \frac{\xi(\theta)^{2}-R^{2}}{\xi(\theta)^{2} R^{2}} \mid d \theta \\
& \leq \frac{C_{4} \varepsilon}{R^{2}} \int_{-\pi}^{\pi}\left|\xi(\theta)^{2}-R^{2}\right| d \theta \\
& \leq C_{5} \varepsilon\left(\beta+\frac{A(N)}{\pi R^{2}}\right) .
\end{aligned}
$$

By (5.13),

$$
\begin{aligned}
\rho^{2} \int_{-\pi}^{\pi}\left(\frac{1}{\xi(\theta)^{2}}-\frac{1}{R^{2}}\right) d \theta & =\rho^{2} \int_{-\pi}^{\pi}\left\{\frac{R^{2}-\xi(\theta)^{2}}{R^{2} \xi(\theta)^{2}}+\frac{\xi(\theta)^{2}-R^{2}}{R^{4}}-\frac{\mu}{R^{2}}\right\} d \theta \\
& =\rho^{2} \int_{-\pi}^{\pi} \frac{\left(R^{2}-\xi(\theta)^{2}\right)^{2}}{R^{4} \xi(\theta)^{2}} d \theta-\frac{2 \pi \mu \rho^{2}}{R^{2}} \\
& \geq \frac{-2 A(N) \rho^{2}}{R^{4}} .
\end{aligned}
$$

Putting together (5.23), (5.24), (5.25) and (5.26) we have

$$
B \geq \frac{-2 A(N) \rho^{2}}{R^{4}}-C_{5} \varepsilon\left(\beta+\frac{A(N)}{\pi R^{2}}\right) .
$$


We now estimate $D$. It is easy to see that

(5.28) $\frac{1}{2}\left(\frac{s(\theta)^{2}}{\xi(\theta)^{2}}-\frac{s(\theta)^{2}}{R^{2}}\right)^{2} \leq\left(\frac{\rho^{2}}{R^{2}}-\frac{s(\theta)^{2}}{R^{2}}\right)^{2}+\left(\frac{s(\theta)^{2}}{\xi(\theta)^{2}}-\frac{\rho^{2}}{R^{2}}\right)^{2}$.

Integrating with respect to $\theta$ and using (5.15) (iii) and (5.14), we have

$(5.29) \int_{-\pi}^{\pi}\left(\frac{\rho^{2}-s(\theta)^{2}}{R^{2}}\right)^{2} d \theta \leq \frac{8 \pi \bar{R}_{o}^{4} \varepsilon^{2}}{R^{4}}=8 \pi \varepsilon^{2}\left(\frac{\bar{R}_{o}}{R_{i}} \frac{R_{i}}{\rho} \frac{\rho}{R}\right)^{4} \leq C_{6} \varepsilon^{2}$.

Using Hölder's inequality,

$$
\begin{aligned}
\left(\int_{\{\xi(\theta) \leq R\}} R^{2}-\xi(\theta)^{2} d \theta\right)^{2} & \leq\left(\int_{-\pi}^{\pi}\left|\xi(\theta)^{2}-R^{2}\right| d \theta\right)^{2} \\
& \leq 2 \pi \int_{-\pi}^{\pi}\left(\xi(\theta)^{2}-R^{2}\right)^{2} d \theta
\end{aligned}
$$

Next

$$
\begin{aligned}
\int_{-\pi}^{\pi}\left(\frac{s(\theta)^{2}}{\xi(\theta)^{2}}-\frac{s(\theta)^{2}}{R^{2}}\right)^{2} d \theta & =\int_{-\pi}^{\pi} \frac{s(\theta)^{4}}{\xi(\theta)^{4} R^{4}}\left(R^{2}-\xi(\theta)^{2}\right)^{2} d \theta \\
& \geq \inf \frac{s(\theta)^{4}}{\xi(\theta)^{4} R^{4}} \int_{-\pi}^{\pi}\left(R^{2}-\xi(\theta)^{2}\right)^{2} d \theta
\end{aligned}
$$

First employing (5.8) in (5.30) and (5.15) (ii) in (5.31), and then combining the two estimates we see that

$$
\int_{-\pi}^{\pi}\left(\frac{s(\theta)^{2}}{\xi(\theta)^{2}}-\frac{s(\theta)^{2}}{R^{2}}\right)^{2} d \theta \geq C_{7}\left(\beta-\frac{A(N)}{\pi R^{2}}\right)^{2} .
$$

Thus (5.28), together with the estimates in (5.29) and (5.32), yields

$$
D=\int_{-\pi}^{\pi}\left(\frac{s(\theta)^{2}}{\xi(\theta)^{2}}-\frac{\rho^{2}}{R^{2}}\right)^{2} d \theta \geq C_{8} \beta^{2}-C_{9} \varepsilon^{2}-C_{10} \frac{A(N)}{\pi R^{2}}
$$

By the assumptions in (5.14) and the conclusions of (5.15), the positive constants $C_{1}-C_{10}$ are either absolute or depend only on $\delta$. The estimates in (5.18), (5.27) and (5.33) when used in (5.22) yield the estimate of the lemma, namely,

$$
I \geq \frac{2 \pi}{\log R / \rho}+K_{0} \beta^{2}-K_{1} \varepsilon \beta-K_{2} \varepsilon^{2}
$$

where $K_{0}, K_{1}, K_{2}$, are positive constants depending only on $\delta$ and become absolute once a value for $\delta$ is chosen. 
Remark 5.1: We intend to use Lemma 5.1 with $F(T)=O, F\left(t_{0}\right)=Q$ and $x_{i}=0$ (see Section 4 and (4.15)). It is easily verified, with $\varepsilon=$ $6 \sqrt{k} \alpha$ (see (4.9)) and $\delta=0.11$, that (4.14) together with (2.6) implies (5.14). Thus, with $\beta=A\left(F\left(t_{0}\right) \backslash B\left(x_{i}, R\right)\right) / A\left(t_{0}\right) \geq \alpha / 2$ (see (4.13)), it is easily seen that there are absolute constants $K>0$ and $k_{1}>0$ such that for $k \leq k_{1}$ we have the following lower bound in (4.15), namely,

$$
\operatorname{Cap}(\Gamma) \geq \frac{2 \pi}{\log R / \rho}+K \alpha^{2}
$$

In Section 6, we work out the proof of Theorem 1 in Case 2.

\section{Proof of Theorem 1 in Case 2}

By (4.1), it follows that

$$
\frac{1}{2} \log \frac{A(\Omega)}{A(T)}=\frac{1}{2} \log \frac{\pi}{A(T)} \geq \frac{1}{2} \log \frac{A\left(t_{0}\right)}{A(T)}=\log \frac{R}{\rho} .
$$

Thus it is seen from (6.1), (5.34) and (4.15) with $v=\left(G-t_{0}\right) /\left(T-t_{0}\right)$ (see the last paragraph of $\S 4$ ) and $k \leq k_{1}$, that

$$
\begin{aligned}
(6.2) \int_{F\left(t_{0}\right) \backslash \bar{F}(T)}|D G|^{2} d x d y & =\left(T-t_{0}\right)^{2} \operatorname{Cap}_{2}(\Gamma) \\
& \geq\left(T-t_{0}\right)^{2}\left\{\frac{4 \pi}{\log (\pi / A(T))}+K \alpha^{2}\right\} .
\end{aligned}
$$

We now estimate $t_{0}$ in (4.1); recall that $G(x)=t_{0}$ on $\partial F\left(t_{0}\right)$ (also see (4.2)). Let $\bar{\Gamma}$ be the condenser $\bar{\Gamma}\left(\bar{F}\left(t_{0}\right), \mathbb{R}^{2} \backslash \Omega\right)$. By symmetrization $[4$; p. 3] we find

$$
\operatorname{Cap}_{2}(\bar{\Gamma})=\frac{1}{t_{0}^{2}} \int_{\Omega \backslash \bar{F}\left(t_{0}\right)}|D G|^{2} d x d y \geq \frac{4 \pi}{\log \left(\pi / A\left(t_{0}\right)\right)}
$$

It follows from (4.1) that

$$
t_{0}^{2} \leq \frac{1}{4 \pi} \log \frac{1}{1-k \alpha^{2}} \int_{\Omega \backslash \bar{F}\left(t_{0}\right)}|D G|^{2} d x d y .
$$

An application of the divergence theorem yields

$$
\begin{aligned}
\int_{\Omega \backslash \bar{F}\left(t_{0}\right)}|D G|^{2} d x d y & =t_{0} \int_{\partial F\left(t_{0}\right)} \frac{\partial G}{\partial n}=-t_{0} \int_{|x|=r} \frac{\partial G}{\partial r} \\
& =2 \pi r t_{0}\left(\frac{1}{r}+O(1)\right) \rightarrow 2 \pi t_{0} \text { as } r \rightarrow 0^{+}
\end{aligned}
$$


Employing (6.4) in (6.3) and noting (2.6), we find that

$$
t_{0} \leq k \alpha^{2} \equiv M
$$

We see from (6.2), (4.13) and (2.9) that there is an absolute constant $C$ such that

$$
\begin{aligned}
\int_{\Omega \backslash \bar{F}(T)}|D G|^{2} d x d y & \geq \int_{F\left(t_{0}\right) \backslash \bar{F}(T)}|D G|^{2} d x d y \\
& \geq \frac{4 \pi\left(T-t_{0}\right)^{2}}{\log (\pi / A(T))}\left(1+C \alpha^{2}\right) .
\end{aligned}
$$

It is easy to check that (see (6.4)),

$$
\int_{\Omega \backslash \bar{F}(T)}|D G|^{2} d x d y=2 \pi T .
$$

We study two cases, namely, (i) $T>M$ and (ii) $T \leq M$, where $M$ is as in (6.5).

Case(i): Let $T>M$. Then from (6.6) and (6.7) we find that

$$
\frac{4 \pi\left(T-k \alpha^{2}\right)^{2}}{\log (\pi / A(T))}\left(1+C \alpha^{2}\right) \leq 2 \pi T .
$$

Simplifying,

$$
\left(T-k \alpha^{2}\right)^{2} \leq \frac{T}{1+C \alpha^{2}} \frac{1}{2} \log \frac{\pi}{A(T)} .
$$

On expanding the left hand side and simplifying (we may take $C<1$ ), we have

$$
T \leq \frac{1}{2}\left(1-\frac{C}{2} \alpha^{2}\right) \log \frac{\pi}{A(T)}+2 k \alpha^{2} .
$$

Recalling (2.9) we see that by taking $k \leq k_{2}, k_{2}$ small enough, (6.8) yields

$$
T \leq \frac{1}{2}\left(1-\bar{C} \alpha^{2}\right) \log \frac{\pi}{A(T)}
$$

where $\bar{C}$ is an absolute constant. Again with $\rho=\sqrt{A(T) / \pi} \leq \sqrt{0.9}$, we get from (6.9) that

$$
T \leq\left(1-\bar{C} \alpha^{2}\right) \log \frac{1}{\rho}
$$


i.e.,

$$
G^{*}(\rho) \leq\left(1-\bar{C} \alpha^{2}\right) \log \frac{1}{\rho} .
$$

We proceed now as in Section 3. We apply the usual isoperimetric inequality to obtain (see (3.2) and (3.3))

$$
\frac{d t}{d A} \geq-\frac{1}{2 A}
$$

For $t>T$,

$$
t-T \leq \frac{1}{2} \log \frac{A(T)}{A(t)} .
$$

With $r=\sqrt{A(t) / \pi}$, we find

$$
G^{*}(r)-G^{*}(\rho) \leq \log \frac{\rho}{r}
$$

Combining (6.10) and (6.11), for $0<r<\sqrt{0.8} \leq \sqrt{A(T) / \pi}$, we deduce that

$$
\begin{aligned}
G^{*}(r) & \leq \log \frac{\rho}{r}+\left(1-\bar{C} \alpha^{2}\right) \log \frac{1}{\rho} \\
& =\log \frac{1}{r}-\bar{C} \alpha^{2} \log \frac{1}{\rho} .
\end{aligned}
$$

Recalling (2.9) we obtain

$$
G^{*}(r) \leq \log \frac{1}{r}-\tilde{C} \alpha^{2}, \quad 0<r<\sqrt{0.8} .
$$

Thus (6.12) implies Theorem 1 in Case (i).

Case (ii): We now consider the case $T \leq M$, where $M$ is as in (6.5). Again, (6.11) holds for $t>T$, i.e., for $0<r<\rho$. Thus

$$
\begin{aligned}
t \leq \log \frac{\rho}{r}+T & \leq \log \frac{\rho}{r}+k \alpha^{2} \\
& =\log \frac{1}{r}-\log \frac{1}{\rho}+k \alpha^{2} \\
& \leq \log \frac{1}{r}-\frac{1}{2} \log \frac{1}{0.9}+k \alpha^{2} .
\end{aligned}
$$

Once again the estimate (2.5) in Theorem 1 holds for $k$ small.

Thus Theorem 1 is completely proven for domains $\Omega$ that are simply connected. 


\section{The multiply connected case.}

We now present details of the proof of Theorem 1 when $\Omega$ is multiply connected. The proof in Case 1 undergoes no change. However, as the study of the geometry of sets in Case 2 involves the use of Bonnesen inequalities, we need to modify the calculations somewhat. The basic aim is to ensure that the estimates of Section 4 continue to hold, with minor modifications, and that the geometry of the condenser $\Gamma$ is not significantly altered (see (4.15)). Thus the result of Lemma 5.1 can be applied even when $\Omega$ is multiply connected. This will in turn ensure that the analysis in Section 6 continues to hold thus yielding the estimate (2.5) in Case 2.

Let us then begin by recalling the definitions of $T$ and $t_{0}$ (see (2.9), (2.10), (4.1) and (4.2)). Firstly, $T$ is such that

$$
0.8 \pi \leq A(T) \leq 0.9 \pi
$$

and

$$
L(\partial F(T))^{2}<4 \pi\left(1+k \alpha^{2}\right) A(T) .
$$

Secondly, $t_{0}$ is such that

$$
\left(1-k \alpha^{2}\right) \pi \leq A\left(t_{0}\right) \leq \pi
$$

and

$$
L\left(\partial F\left(t_{0}\right)\right)^{2}<4 \pi(1.01) A\left(t_{0}\right)
$$

Noting that Green's function $G(x)$ has only one pole in $\Omega$, it follows from the maximum principle that $F(t)$ has only one component. However, in general, $F(t)$ would be multiply connected except, perhaps when $t$ is large. With some abuse of notation, let $H(t)$ denote the holes of $F(t)$, i.e., the set $F(t) \cup H(t)$ is simply connected. Since $T>t_{0}$, $F(T) \subset F\left(t_{0}\right)$. We now prove a lemma that will provide us with an estimate for the perimeter and the area of the holes $H(T)$ of $F(T)$.

Lemma 7.1: Let $D \subset \mathbb{R}^{2}$ be a bounded domain whose boundary $\partial D$ consists of a finite union of rectifiable curves. Let $H$ denote the holes of $D$, i.e., the set $D \cup H$ is simply connected. Suppose that for some small $\delta>0$, we have

$$
L(\partial D)^{2} \leq 4 \pi(1+\delta) A(D)
$$


Then

$$
L(\partial H) \leq \frac{\delta}{2} L(\partial D) \text { and } A(H) \leq \frac{\delta^{2}}{4} A(D)
$$

Proof: Let $S=D \cup H$; then $L(\partial S)+L(\partial H)=L(\partial D)$, and $A(S)=$ $A(D)+A(H)$. Thus,

$$
L(\partial D)^{2}=\{L(\partial S)+L(\partial H)\}^{2} \leq 4 \pi(1+\delta) A(D) .
$$

Expanding, we obtain

$$
L(\partial S)^{2}+2 L(\partial S) L(\partial H)+L(\partial H)^{2} \leq 4 \pi(1+\delta) A(D) .
$$

Applying the usual isoperimetric inequality for $S$ and $H$, employing $A(D) \leq A(S)$ and simplifying (7.6), we see that

$$
2 \sqrt{(A(D)+A(H)) A(H)}+A(H) \leq \delta A(D) .
$$

Thus,

$$
A(H) \leq \frac{\delta^{2}}{4} A(D) .
$$

Again using $A(D) \leq A(S)$, (7.6) yields

$$
2 L(\partial S) L(\partial H)+L(\partial H)^{2} \leq 4 \pi \delta A(S) \leq \delta L(\partial S)^{2} .
$$

Finally,

$$
L(\partial H) \leq \frac{\delta}{2} L(\partial S) \leq \frac{\delta}{2} L(\partial D)
$$

Thus (7.5) holds.

Our strategy for multiply connected domains is as follows. Set $B=F(T) \cup H(T)$ and $H=B \cap H\left(t_{0}\right)$, i.e., $H$ denotes the holes of $F\left(t_{0}\right)$ that lie in $B$. Note that $H \subset H(T)$. It is clear that $B$ is simply connected. Also set $D=H \cup F\left(t_{0}\right)$. The motivation for this choice of $H$ follows from the observation that (7.2) and Lemma 7.1 imply that $A(H) \leq A(H(T)) \leq k^{2} \alpha^{4}$ (see (7.8)). However, it is not clear that $A\left(H\left(t_{0}\right)\right)$ can be bounded by such a term (see (7.4)). With these modifications, we employ the methods of Section 4 to describe the geometry of the condenser $\Gamma^{\prime}=\Gamma^{\prime}\left(\bar{B}, \mathbb{R}^{2} \backslash D\right)$ and conclude (4.14). Now, if $\Gamma$ is the condenser $\Gamma\left(\bar{F}(T), \mathbb{R}^{2} \backslash F\left(t_{0}\right)\right)$ then $\operatorname{Cap}(\Gamma) \geq \operatorname{Cap}\left(\Gamma^{\prime}\right)$. We use Lemma 5.1 to get a lower bound for $\operatorname{Cap}\left(\Gamma^{\prime}\right)$. The desired estimate for $\operatorname{Cap}(\Gamma)$ will then be shown to follow from this estimate for $\operatorname{Cap}\left(\Gamma^{\prime}\right)$. 
We now proceed with the calculations to estimate the inradius and outradius of the set $B=F(T) \cup H(T)$. Recall (7.1) and (7.2). By an application of Lemma 7.1,

$$
A(H(T)) \leq \frac{k^{2} \alpha^{4}}{4} A(T) \text { and } L(\partial H(T)) \leq \frac{k \alpha^{2}}{2} L(\partial F(T)) .
$$

Call $S=\partial F(T) \backslash \partial H(T)=\partial B$. Note that $L(\partial B)=L(S) \leq L(\partial F(T))$ and $A(T) \leq A(B)$. If $R_{i}$ and $R_{o}$ denote the inradius and the outradius of $B$ respectively then (2.11), (2.12), (2.13), (7.1), (7.2) and (7.8) yield $\pi^{2}\left(R_{o}-R_{i}\right)^{2} \leq L(\partial B)^{2}-4 \pi A(B) \leq L(\partial F(T))^{2}-4 \pi A(T) \leq 4 \pi k \alpha^{2} A(T)$.

Thus,

$$
0 \leq R_{o}-R_{i} \leq 2 \sqrt{\frac{k A(T)}{\pi}} \alpha \leq 2 \sqrt{k} \alpha
$$

Also,

$$
\begin{aligned}
R_{o} & \leq \frac{1}{2 \pi}\left\{L(\partial B)+\sqrt{L(\partial B)^{2}-4 \pi A(B)}\right\} \\
& \leq \frac{1}{2 \pi}\left\{L(\partial F(T))+\sqrt{L(\partial F(T))^{2}-4 \pi A(T)}\right\} \\
& \leq \frac{1}{2 \pi} L(\partial F(T))+\alpha \sqrt{\frac{k A(T)}{\pi}} \\
& \leq(1+2 \sqrt{k} \alpha) \sqrt{\frac{A(T)}{\pi}}
\end{aligned}
$$

and using the usual isoperimetric inequality for $F(T)$, we see

$$
\begin{aligned}
R_{i} & \geq \frac{1}{2 \pi}\left\{L(\partial B)-\sqrt{L(\partial B)^{2}-4 \pi A(B)}\right\} \\
& \geq \frac{1}{2 \pi}\left\{L(\partial F(T))-L(\partial H(T))-\sqrt{L(\partial F(T))^{2}-4 \pi A(T)}\right\} \\
& \geq\left\{\left(1-k \alpha^{2} / 2\right)-\sqrt{k} \alpha\right\} \sqrt{\frac{A(T)}{\pi}} \\
(7.11) & \geq(1-1.05 \sqrt{k} \alpha) \sqrt{\frac{A(T)}{\pi}} .
\end{aligned}
$$

Clearly, there exist $x_{o}$ and $x_{i}$ in $\mathbb{R}^{2}$ such that $S$ is contained in the region between the two circles $C_{o}=\left\{x:\left|x-x_{o}\right|=R_{o}\right\}$ and $C_{i}=\{x$ : $\left.\left|x-x_{i}\right|=R_{i}\right\}$. It is easy to see from (7.9) that

$$
\left|x_{o}-x_{i}\right| \leq R_{o}-R_{i} \leq 2 \sqrt{k} \alpha .
$$


Set

$$
\varepsilon=6.5 \sqrt{k} \alpha
$$

It is necessary to estimate the outradius $\hat{R}_{o}$ of $F\left(t_{0}\right)$, which is same as the outradius of $F\left(t_{0}\right) \cup H\left(t_{0}\right)$. From (7.4) and Lemma 7.1, we see that $A\left(H\left(t_{0}\right)\right) \leq 0.0001 A\left(t_{0}\right)$ and $L\left(\partial F\left(t_{0}\right)\right) \leq 0.005 L\left(\partial F\left(t_{0}\right)\right)$. Set $E=F\left(t_{0}\right) \cup H\left(\overline{t_{0}}\right)$. Using $L(\partial E) \leq L\left(\partial F\left(t_{0}\right)\right), \bar{A}\left(t_{0}\right) \leq A(E)$ and (7.4) in (2.12), we find

$$
\begin{aligned}
\hat{R}_{o} & \leq \frac{1}{2 \pi}\left\{L(\partial E)+\sqrt{L(\partial E)^{2}-4 \pi A(E)}\right\} \\
& \leq \frac{1}{2 \pi}\left\{L\left(\partial F\left(t_{0}\right)+\sqrt{L\left(\partial F\left(t_{0}\right)^{2}-4 \pi A\left(t_{0}\right)\right.}\right\}\right. \\
& \leq\{\sqrt{1.01}+\sqrt{0.01}\} \sqrt{\frac{A\left(t_{0}\right)}{\pi}} \\
& \leq 1.11 \sqrt{\frac{A\left(t_{0}\right)}{\pi}} .
\end{aligned}
$$

Let $H=B \cap H\left(t_{0}\right)$, i.e., $H$ denotes the holes of $F\left(t_{0}\right)$ which lie in $B$. Clearly, $H \subset H(T)$, and (7.8) implies

$$
A(H) \leq A(H(T)) \leq \frac{k^{2} \alpha^{4}}{4} A(T) \leq \frac{k^{2} \alpha^{4}}{4} A\left(t_{0}\right) \leq \frac{\pi}{4} k^{2} \alpha^{4}
$$

The set $H$ need not contain all the holes of $F\left(t_{0}\right)$, and so $\left(\mathbb{R}^{2} \backslash B\right) \cap H\left(t_{0}\right)$ may be nonempty. Now set $R=\sqrt{A\left(t_{0}\right) / \pi}, \rho=\sqrt{A(T) / \pi}$ and $D=$ $F\left(t_{0}\right) \cup H$. Define

$$
R^{\prime}=\sqrt{\frac{A(D)}{\pi}}, \rho^{\prime}=\sqrt{\frac{A(B)}{\pi}}, \text { and } \beta^{\prime}=\frac{A\left(D \backslash B\left(x_{i}, R^{\prime}\right)\right)}{A(D)}
$$

where $x_{i}$ is as in (7.12). In the event that $\Omega$ is multiply connected, $x_{o}$ may not lie in $F\left(t_{0}\right)$. Observe that (7.1), (7.3) and (7.14) imply the following, namely,

$$
\begin{aligned}
\sqrt{0.8} \leq \rho \leq \rho^{\prime} & =\sqrt{\frac{A(B)}{\pi}}=\sqrt{\frac{A(T)+A(H(T))}{\pi}} \\
& \leq \rho \sqrt{1+\left(k^{2} \alpha^{4}\right) / 4}
\end{aligned}
$$

and

$$
\begin{aligned}
\sqrt{0.999} \leq \sqrt{1-k \alpha^{2}} & \leq R \leq R^{\prime}=\sqrt{\frac{A\left(t_{0}\right)+A(H)}{\pi}} \\
& \leq R \sqrt{1+\left(k^{2} \alpha^{4}\right) / 4}
\end{aligned}
$$


We may now carry out the calculations in (4.10), (4.11) and conclude (4.14) (i) - (v) for the sets $B$ and $D$. We list these for easy reference. First note that (i) $B\left(x_{i}, R_{i}\right) \subset B \subset B\left(x_{i}, \bar{R}_{o}\right)$, where $\bar{R}_{o} \leq$ $2 R_{o}-R_{i} \leq(1+5.05 \sqrt{k} \alpha) \rho \leq 1.12$, and (ii) $B \subset \bar{B} \subset D \subset B\left(x_{i}, \tilde{R}_{o}\right)$, where $\tilde{R}_{o} \leq 2 \hat{R}_{o}-R_{i} \leq 2.22 R-(1-1.05 \sqrt{k} \alpha) \rho \leq 1.40$.

Furthermore, (a) $R_{i} \leq \rho^{\prime} \leq \bar{R}_{o}$ and $R^{\prime} \leq \hat{R}_{o} \leq \tilde{R}_{o}$, (b) $\bar{R}_{o}-R_{i} \leq$ $2\left(R_{o}-R_{i}\right) \leq 4 \sqrt{k} \alpha \leq \varepsilon$, where $\varepsilon=6.5 \sqrt{k} \alpha$, (c) $(1-\varepsilon) \leq(1-$ $6.1 \sqrt{k} \alpha) \leq R_{i} / \bar{R}_{o} \leq \rho^{\prime} / \bar{R}_{o} \leq 1,(\mathrm{~d}) 4 / 7 \leq(1-1.05 \sqrt{k} \alpha) \rho / 1.40 \leq$ $R_{i} / \tilde{R}_{o} \leq \rho^{\prime} / \tilde{R}_{o} \leq \rho^{\prime} / R^{\prime} \leq \sqrt{0.9\left(1+k^{2} \alpha^{4} / 4\right) / 0.999} \leq \sqrt{10001 / 11100}$

Let $\Gamma=\Gamma\left(\bar{F}(T), \mathbb{R}^{2} \backslash F\left(t_{0}\right)\right)$ denote the condenser consisting of the pair $\bar{F}(T)$ and $\mathbb{R}^{2} \backslash F\left(t_{0}\right)$, and let $\Gamma^{\prime}$ be the condenser $\Gamma\left(\bar{B}, \mathbb{R}^{2} \backslash D\right)$. Then

$$
\operatorname{Cap}(\Gamma) \geq \operatorname{Cap}\left(\Gamma^{\prime}\right)
$$

Now let $\beta^{\prime}$ be as in (7.15). We will now derive a lower bound for $\operatorname{Cap}(\Gamma)$ of the type given by Lemma 5.1 by first calculating a lower bound for $\operatorname{Cap}\left(\Gamma^{\prime}\right)$. The final estimate for $\operatorname{Cap}(\Gamma)$ will follow from (7.18). We apply now the symmetrization of Section 5 to the set $D$, relative to $B\left(x_{i}, R^{\prime}\right)$. Taking $\delta=0.1$ we may verify (5.14) and (5.15) for $\beta^{\prime}, \varepsilon, \rho^{\prime}, R^{\prime}, R_{i}, \bar{R}_{o}$ and $\tilde{R}_{o}$. Thus we may conclude the estimate in Lemma 5.1 for $\operatorname{Cap}\left(\Gamma^{\prime}\right)$, namely,

$$
\operatorname{Cap}\left(\Gamma^{\prime}\right) \geq \frac{2 \pi}{\log \left(R^{\prime} / \rho^{\prime}\right)}+K_{1} \beta^{2}-K_{2} \varepsilon^{2}-K_{3} \varepsilon \beta^{\prime},
$$

where $K_{1}, K_{2}$, and $K_{3}$ are absolute constants. Our intention is to express the right hand side of (7.19) in terms of $\rho, R$, and $\alpha$. By (7.16) and (7.17), we see that

$$
\log \frac{R^{\prime}}{\rho^{\prime}} \leq \log \frac{R \sqrt{1+\left(k^{2} \alpha^{4}\right) / 4}}{\rho} \leq \log \frac{R}{\rho}+\frac{k^{2} \alpha^{4}}{8} .
$$

Recalling that $\sqrt{0.8} \leq \rho \leq \sqrt{0.9}, \sqrt{0.999} \leq R \leq 1$ (see (7.1) and (7.3)) and $\log (1+x) \geq 2 x / 3(0<x<0.5)$, we see from (7.17) that for $k<k_{0}$, small, there is an absolute constant $K_{4}$ such that

$$
\frac{2 \pi}{\log \left(R^{\prime} / \rho^{\prime}\right)} \geq \frac{2 \pi}{\log (R / \rho)}-K_{4} k^{2} \alpha^{4} .
$$

Recall that the quantity $\beta$, in (4.12), is bounded below by $\alpha / 2$ (see (4.13)). We now estimate $\beta^{\prime}$; set $\bar{R}=\sqrt{A(\Omega \cup H) / \pi}>1$. Note that 
$D \subset \Omega \cup H$ and so $\bar{R}>R^{\prime}$; thus (1.1), (7.1) and (7.14) yield

$$
\begin{aligned}
\beta^{\prime} & =\frac{A\left(D \backslash B\left(x_{i}, R^{\prime}\right)\right)}{A(D)} \\
& \geq \frac{A\left((\Omega \cup H) \backslash B\left(x_{i}, R^{\prime}\right)\right)-A((\Omega \cup H) \backslash(D))}{A(\Omega \cup H)} \\
& \geq \frac{A\left(\Omega \backslash B\left(x_{i}, \bar{R}\right)\right)-A\left(\Omega \backslash F\left(t_{0}\right)\right)}{B\left(x_{i}, \bar{R}\right)} \\
& \geq \frac{A\left(\Omega \backslash B\left(x_{i}, 1\right)\right)-A\left(B\left(x_{i}, \bar{R}\right) \backslash B\left(x_{i}, 1\right)\right)-A\left(\Omega \backslash F\left(t_{0}\right)\right)}{\pi\left(1+k^{2} \alpha^{4}\right)} \\
(7.22) & \geq \frac{\alpha-k^{2} \alpha^{4}-k \alpha^{2}}{1+k^{2} \alpha^{4}} \geq \alpha / 2,
\end{aligned}
$$

where $k$ is small. This gives us (4.14) (vi) for $\beta^{\prime}$.

It is clear from $(7.18),(7.19),(7.20),(7.21)$ and (7.22) that there is a $k_{1}>0$, and an absolute constant $K>0$, such that

$$
\operatorname{Cap}(\Gamma) \geq \frac{2 \pi}{\log (R / \rho)}+K \alpha^{2}
$$

The rest of the proof now follows from the analysis in Section 6 .

\section{The eigenvalue problem.}

The problem of ascertaining the dependence on asymmetry of the first eigenvalue $\lambda_{1}$ for the Laplacian

$$
\Delta u+\lambda u=0 \quad \text { in } \Omega, \quad u=0 \quad \text { on } \partial \Omega,
$$

seems more difficult than the corresponding problem for capacity, but can be reformulated in terms of the Green's function.

Conjecture: Let $\lambda=\lambda_{1}$ be the first eigenvalue for (8.1), where $\Omega$ is a bounded plane region. Then there exists a constant $C$ such that

$$
\lambda_{1} \geq\left(1+C \alpha(\Omega)^{2}\right) \lambda_{1}^{*},
$$

where $\lambda_{1}^{*}=\lambda\left(\Omega^{*}\right)$ is the first eigenvalue for the disk having the same area as $\Omega$. 
The ellipse shows that this conjecture, if true, is best possible. This can be deduced from the expansion in $[10 ; \mathrm{p} .326]$. At present, the best result is due to Hansen and Nadirashvili [9], who showed that (8.2) is true with exponent 3 in place of 2 .

Using the fact that the solution to (8.1) can be written

$$
u(z)=\frac{\lambda}{2 \pi} \int_{\Omega} G_{\Omega}(z, \zeta) u(\zeta) d \zeta
$$

where $G_{\Omega}$ is the Green's function for $\Omega$, it can easily be shown that (8.2) would follow from the hypothetical inequality

$$
\int_{\Omega} \int_{\Omega} \ldots \int_{\Omega} G_{\Omega}\left(0, \zeta_{1}\right) G_{\Omega}\left(\zeta_{1}, \zeta_{2}\right) \ldots G_{\Omega}\left(\zeta_{n-1}, \zeta_{n}\right) d \zeta_{1} \ldots d \zeta_{n}
$$

$\leq\left(1-c \alpha^{2}\right)^{n} \int_{\Omega^{*}} \int_{\Omega^{*}} \ldots \int_{\Omega^{*}} G_{\Omega^{*}}\left(0, \zeta_{1}\right) G_{\Omega^{*}}\left(\zeta_{1}, \zeta_{2}\right) \ldots G_{\Omega^{*}}\left(\zeta_{n-1}, \zeta_{n}\right) d \zeta_{1} \ldots d \zeta_{n}$.

where $\Omega^{*}$ is the disk of the same area as $\Omega$. The case $n=1$ in (8.3) follows from Theorem 1 . 


\section{References}

1. C. Bandle, Isoperimetric Inequalities and Applications, Pitman Monographs and Studies in Math., 7, Boston, 1980.

2. T. Bhattacharya and A. Weitsman, Bounds for capacities in terms of asymmetry, To appear in Revista Matematica Iberoamericana.

3. H. Federer, Geometric Measure Theory, Springer-Verlag, Berlin, 1969 .

4. H. Federer and W. Fleming, Normal and integral currents, Ann. of Math. 72 (1960).

5. F. Gehring, Inequalities for condensers, hyperbolic capacity, and extremal length, Mich. Math. J. 18 (1971), 1-21.

6. R. Hall, A quantitative isoperimetric inequality in $n$ dimensional space, J. Reine angew. Math 428 (1992), 61-76.

7. R. Hall, W. Hayman and A. Weitsman, On capacity and asymmetry, J. d'Analyse Math. 56(1991), 87-123.

8. W. Hansen and N. Nadirashvili, Isoperimetric inequalities for capacities, Harmonic Analysis and Discrete Potential Theory, ed. M. Picardello, Plenum Press, 1992, 193-206.

9. W. Hansen and N. Nadirashvili, Isoperimetric inequalities in potential theory, Potential Analysis 3(1994), 1-14.

10. D. Joseph, Parameter and domain dependence of eigenvalues of elliptic partial differential equations, Arch. Rat. Mech. Anal. 24 (1967), 326-351.

11. R. Osserman, Bonnesen-style isoperimetric inequalities, Am. Math. Monthly 86(1972), 1-29.

Indian Statistical Institute

7 SJSS Marg

New Delhi 110016

India
Department of Mathematics

Purdue University

W. Lafayette, IN 47907

USA 